\title{
EVOLUCIÓN DEL COMPORTAMIENTO ESTRATÉGICO DE LAS COOPERATIVAS VERSUS EMPRESAS CAPITALISTAS: UNA APROXIMACIÓN DESCRIPTIVA AL EFECTO CRISIS
}

\author{
POR \\ Jesús CLEMENTE LÓPEZ, \\ Millán DÍAZ FONCEA, \\ María A. GONZÁLEZ ÁLVAREZ, \\ Carmen MARCUELLO SERVÓS ${ }^{1} \mathrm{y}$ \\ Pablo NACHAR CALDERÓN ${ }^{2}$
}

\section{RESUMEN}

Desde el año 2008 la situación económica en España ha sufrido un proceso de deterioro que no se había conocido en otros periodos. La situación es tan compleja que es necesario abordar el estudio de numerosas cuestiones a nivel macroeconómico, microeconómico, del sector financiero y de la economía real para comprender y, especialmente, para reconocer qué factores están permitiendo sobrevivir a los proyectos empresariales. En este sentido, dos factores fundamentales para la supervivencia de las organizaciones económicas en la crisis actual son la capacidad de exportación y de innovación. El objetivo de la investigación es ambos fenómenos de las sociedades cooperativas de trabajo y las sociedades laborales en España, comparando su situación con las empresas capitalistas, con el fin de obtener una imagen real del posicionamiento de las Empresas de Economía Social en estos ámbitos. Los datos utilizados para realizar el estudio proceden de la Encuesta sobre Estrategias Empresariales (ESEE) para el periodo 2005-2010, y se realiza atendiendo especialmente a los sectores.

\footnotetext{
1 Profesores de la Universidad de Zaragoza. Direcciones de correo electrónico: clemente@unizar.es, millan@unizar.es, mara.gonzalez@esisl.com y cmarcue@unizar.es.

2 Profesor de la Universidad Diego Portales (Santiago, Chile). Dirección de correo electrónico: pablo.nachar@mail.udp.cl.
}

REVESCO No 116 - Tercer Cuatrimestre 2014 - ISSN: 1885-8031 - www.ucm.es/info/revesco

http://dx.doi.org/10.5209/rev_REVE.2014.v116.45718

Fecha de recepción: 23/07/2013

Fecha de aceptación: 16/06/2014 
Palabras clave: sociedades cooperativas, exportación, innovación, análisis comparativo, España.

Claves Econlit: J21, M20

EVOLUTION OF STRATEGIC BEHAVIOR OF COOPERATIVE VERSUS CAPITALIST ENTERPRISES: A DESCRIPTIVE APPROACH TO EFFECTIVE CRISIS

\begin{abstract}
Since 2008 the economic situation in Spain has undergone a process of deterioration. The situation is so complex that it is necessary to study it of numerous perspectives because it is very important to know the factors that are enabling firms survive. In this regard, two key factors in this topic are the ability to export and innovation. The aim of the paper it is to analyse both phenomena in Social Firms Spain, comparing their situation with capitalist enterprises, in order to get a picture of the position of Social Economy Enterprises in these areas. The data used for the study come from the Survey on Business Strategies (ESEE) for the period 2005-2010.
\end{abstract}

Keywords: cooperatives, export, innovation, comparative analysis, Spain.

\title{
1. INTRODUCCIÓN
}

Desde el año 2008 la situación económica en España ha sufrido un proceso de deterioro que no se había conocido en otros periodos. La situación es tan compleja que es necesario abordar el estudio de numerosas cuestiones a nivel macroeconómico, microeconómico, del sector financiero y de la economía real para comprender y, especialmente, para reconocer qué factores están permitiendo sobrevivir a los proyectos empresariales.

En el conjunto de la Unión Europa, España junto con Italia, Francia y Alemania, son los países con un sector de la Economía Social más relevante, y en especial, si se tienen en cuenta a las sociedades cooperativas de trabajo y otras organizaciones asimiladas como las sociedades laborales. En algunos trabajos se encuentra evidencia de la capacidad de las cooperativas para mantener el empleo en este periodo de crisis, su mayor capacidad para remunerar en mejores condiciones a determinados sectores de población frente a la empresa 
capitalista tradicional, así como una mayor reacción medida a través de la creación de un mayor número de empresas (Clemente et al., 2009, 2012). Sin embargo, la coyuntura económica ha provocado que ya no sea suficiente con la capacidad de adaptación y flexibilidad que han demostrado las cooperativas, sino que es necesario abordar otros aspectos que serán claves para enfrentarse a un futuro todavía incierto.

En este sentido, existen cuestiones fundamentales relativas tanto a la gestión interna como a la capacidad de financiación, la planificación, la calidad de los productos y la productividad que se quedan cortos ante el deterioro que causa la situación de crisis para el conjunto de la sociedad. De todas formas, aunque la mejora del entorno general parece estar todavía muy alejada, existe un cierto consenso en que las organizaciones económicas que están superando la situación de crisis son aquéllas que han apostado por la innovación y la exportación. Estos dos aspectos siempre han sido claves, más aún en este momento.

Con todo ello, el objetivo general de este artículo, es describir la capacidad de innovación y de exportación de las sociedades cooperativas de trabajo y las sociedades laborales en España, al entender que éstos son dos factores fundamentales para la supervivencia de las organizaciones económicas en la crisis actual, desde una perspectiva comparativa, es decir, utilizando las otras formas organizativas como elemento de referencia. Este objetivo general se subdivide en dos referidos a las siguientes cuestiones: 1) Examinar las actividades tecnológicas de las cooperativas de trabajo y las sociedades laborales, atendiendo especialmente a los resultados de la innovación que realizan en el periodo 20052010 (esta innovación se divide en innovación en producto, innovación en métodos organizativos e innovaciones comerciales); 2) Estudiar la evolución de las actividades de exportación y características de los mercados en los que operan las cooperativas de trabajo y las sociedades laborales para el mismo periodo de tiempo.

Los datos utilizados para realizar el estudio proceden de la Encuesta sobre Estrategias Empresariales (ESEE): una encuesta anual realizada por la Fundación SEPI que tiene como finalidad "la caracterización del comportamiento estratégico de las empresas manufactureras españolas"3. Esta encuesta utiliza como población de referencia las empresas con 10 o más trabajadores de la industria manufacturera (códigos 10 a 32 de la CNAE-2009 excluido el 19), manteniendo una muestra representativa de las empresas industriales manufactureras en

\footnotetext{
${ }^{3}$ Fundación SEPI (2012): Las Empresas Industriales en 2010, http://www.fundacionsepi.es/esee/sp/sesee informes.asp
} 
España. Esto permite poder comparar los resultados obtenidos por las sociedades cooperativas con el resto de empresas industriales incluidas en la ESEE publicada por la Fundación SEPI.

El trabajo consta de los siguientes apartados: en la sección 2 se revisa la situación de las sociedades cooperativas en relación a su carácter innovador y exportador; en la tercera se presentan los datos del estudio y el análisis descriptivo de los mismos; en las secciones 4 y 5 se muestran los resultados para la actividad exportadora e innovadora, respectivamente y el 6 presenta las principales conclusiones.

\section{INNOVACIÓN Y EXPORTACIÓN EN LAS COOPERATIVAS: UNA REVISIÓN}

Es aceptado que las Cooperativas de Trabajo y Sociedades Laborales (representantes principales de las Empresas de Economía Social) resisten mejor una coyuntura económica desfavorable que otros modelos organizativos. Sin embargo, estas organizaciones no son ajenas a la evolución del mercado y también necesitan plantear una estrategia adecuada para ser viables y mantener su actividad. En este sentido, la innovación y las exportaciones se han mostrado como una forma de afrontar este escenario al ser instrumentos clave para impulsar la productividad de las empresas (OCDE, 2011) y así obtener ventajas competitivas. En este apartado se comentan algunos trabajos previos relacionados con estas dos dimensiones de la dirección estratégica en las entidades de economía social en general y en las cooperativas en particular.

\subsection{Capacidad innovadora y las Empresas de Economía Social}

Según el Manual de Oslo (OCDE, 2005), la innovación abarca la I+D, las actividades de desarrollo de introducción de innovaciones que no son de producto o de proceso (nuevos métodos de comercialización u organizativos) y las adquisiciones de conocimiento externo o de bienes de capital, siendo, por tanto, un concepto amplio que incluye diferentes dimensiones relacionadas con la misma (Echevarría, 2008) y que ha sido analizada desde estas diferentes perspectivas por diversos autores (Damanpòur y Gopalakrishnana, 2001; Koschtzky, 2002; Chesbrough, 2004, 2005; Teo, 2007).

En la revisión de los trabajos realizados sobre la capacidad innovadora de las Empresas de la Economía Social (EES) se observa que han recibido una clara atención en España y a nivel internacional, pero no de forma conjunta. Desde el punto de vista de la innovación, en España se han centrado principalmente en el sector agroalimentario. Por 
ejemplo, Segovia y Sánchez (2010) clasifican las fuentes de innovación de las cooperativas agrarias, atendiendo a sus características estructurales y operativas. Sus resultados evidencian que existen cuatro fuentes de innovación principales (los gerentes, la tecnología en sí misma, el mercado y el contexto normativo) que, intermediados por la orientación al cambio de las áreas de gestión, motivan las innovaciones (internas y externas) en estas organizaciones.

Asimismo, desde el punto de vista industrial, Coque y Pérez (1999) y Coque (2002) analizan la innovación tecnológica de las empresas de participación como uno de los factores clave de competitividad de las empresas actuales. En concreto, Coque (2002) plantea que las sociedades cooperativas muestran diferentes debilidades para desarrollar actividades fuertemente innovadoras, presentando una menor productividad y calidad en la I+D que realizan, debido a la escasez de recursos financieros, a la baja formación de los socios cooperativistas, o a que muchas de ellas realizan actividades tecnológicamente simples. No obstante, esto está cambiando, y como también apunta Coque (2002), aquellas cooperativas en las que la participación esté presente y basen en mayor medida su actividad en los principios cooperativos de la Alianza Cooperativa Internacional, estas debilidades son más fácilmente subsanables, pudiendo convertirse en fortalezas, incluso (p. 10).

En términos generales sobre las cooperativas, Águila y Padilla (2010) inciden en los factores que favorecen la innovación y su relación con el comportamiento estratégico de las empresas de Economía Social, aunque son Amat y Perramon (2011) quienes analizan el crecimiento de las cooperativas desde el enfoque de la competitividad. Estos autores demuestran que los principales factores que favorecen el crecimiento de las empresas, incluidas las cooperativas, y por tanto la competitividad de las mismas, son el desarrollo de técnicas de gestión modernas, el fomento de la calidad y la productividad, así como la innovación y la internacionalización de la empresa.

A nivel internacional, cabe destacar especialmente los trabajos de Antoniololi et al. (2011) y Kalmi y Kauhanen (2008) en los que se analiza la relación entre las actividades de innovación y su efecto sobre el bienestar de los trabajadores.

Finalmente, desde los trabajos que analizan la innovación en el sector de las Empresas de Economía Social se ha señalado la importancia de la innovación social como un elemento relevante en el ámbito de la innovación en general y especialmente importante en el sector de la Economía Social. Así, desde las propias publicaciones de la OCDE sobre innovación en 
2005 y en 2010 se produce un cambio sustancial al reconocer que la innovación social es fundamental para el desarrollo de las sociedades. En este sentido, Morales (2012) realiza un análisis del concepto de innovación social y su articulación a través de diferentes organizaciones de la Economía Social. Su conclusión es que el marco organizativo de las cooperativas es una herramienta que facilita las iniciativas innovadoras. En esta misma línea, Sanchís y Campos (2011) insisten en que los factores organizativos son fundamentales para la generación de innovación, siendo elementos consustanciales en el caso de las cooperativas. Asimismo, el trabajo en red es otro mecanismo que favorece la generación de actividades de innovación (Baleren et al., 2004).

\subsection{Actividad exportadora y Empresas de Economía Social}

Por su lado, la exportación de productos también contiene matices relevantes, desde los diferentes lugares geográficos a los que se exporta, el modelo para el acceso a la exportación y el tipo de mercado y su relación con las importaciones realizadas (Navarro, 2002; Theodosious y Leonidou, 2003; Akyol y Akehurst, 2003; Flor et al., 2004).

Con respecto a las actividades de exportación, el número de trabajos que analizan estas actividades en las Empresas de Economía Social (EES) son escasos, destacando el de Fernández et al. (2008) en el que se analizan las estrategias exportadoras, de expansión, competitivas y las estrategias de producto en las cooperativas de Castilla-La Mancha. Asimismo, Fuentes et al. (2011) plantean que la estrategia de exportación y de internacionalización ha sido utilizada por las cooperativas agrarias para enfrentarse a las dificultades presentes en el mundo agrario (atomización de las empresas, etc.).

Estos autores señalan que, según la Confederación de Cooperativas Agrarias de España (2010), en 2009 el 35\% de las cooperativas españolas vendió en otros países de la Unión Europea e incluso el $15 \%$ exporta a otros países no comunitarios. En el caso de las cooperativas de mayor tamaño (las que superan el millón de euros de facturación) la cifra de exportadoras aumenta hasta el 42,20\%, con un promedio de 12,47 millones de euros exportado por empresa, mostrando la relevancia que tiene el tamaño en la capacidad de exportación.

No obstante, Fuentes et al. (2011) concluyen que, según las estadísticas publicadas, la implantación exterior de las cooperativas españolas es escasa, sólo el 7,41\% de las cooperativas de segundo grado se han establecido en el exterior y apenas la mitad en las 
cooperativas de primer grado, uniéndose a Boccherini (2010) en su precisión de que la agroindustria alimentaria española presenta un escaso interés por la internacionalización.

Para finalizar, cabe destacar que tanto el análisis de la innovación como el de las exportaciones se han realizado utilizando principalmente aproximaciones como el análisis de casos o las entrevistas en profundidad. En este sentido, a partir de la revisión de los informes y publicaciones existentes que han utilizado las Encuesta sobre Estrategias Empresariales (ESEE) en España sobre estos temas, podemos afirmar que las Sociedades Cooperativas de Trabajo y las Sociedades Laborales no han sido analizadas de forma específica y comparada con el resto de formas jurídicas, a pesar de las posibilidades que otorga la Encuesta sobre Estrategias Empresariales (ESEE). Por ello, consideramos que dada la relevancia del tema y la necesidad de ampliar el conocimiento sobre este campo, este proyecto tiene un claro interés académico y divulgativo, ya que completa las perspectivas presentadas hasta la fecha.

\section{DATOS BÁSICOS Y ANÁLISIS DESCRIPTIVO}

La base de datos de este trabajo es la Encuesta sobre Estrategias Empresariales (ESEE). Esta encuesta se realiza anualmente a las empresas industriales manufactureras en España y tiene como objetivo "la caracterización del comportamiento estratégico de las empresas manufactureras españolas". Esta encuesta tiene como población de referencia las empresas de la industria manufacturera (códigos 10 a 32 de la CNAE-2009 excluido el 19) con 10 o más trabajadores, por lo que se restringe la presencia de las empresas de menor tamaño. En concreto, la muestra recoge un total de 1.731 empresas, diferenciándolas por tamaño, entre aquéllas de menos de 200 trabajadores (1.364 empresas) y las de más de 200 trabajadores (367 empresas).

La ESEE parte de un cuestionario de 107 preguntas, con más de 500 campos, que incorpora información acerca de las estrategias de las empresas (precios, gastos de I+D...) y de su entorno competitivo (mercados en los que están presente), así como información sobre sus cuentas de resultados y balances contables. Entre las fortalezas de la ESEE se incluyen la consistencia y calidad de la serie al mantener a lo largo del tiempo las mismas empresas encuestadas, incorporando empresas con características similares a las existentes cuando éstas son baja en la muestra (ya sea por causa voluntaria u obligada por su desaparición, etc.). Asimismo, en la selección inicial de empresas realizada en 1999 se combinaron criterios de

\footnotetext{
${ }^{4}$ Fundación SEPI (2012): Las Empresas Industriales en 2010, http://www.fundacionsepi.es/esee/sp/sesee_informes.asp
} 
exhaustividad y de muestreo aleatorio con el fin de mantener el nivel de representatividad del sector manufacturero. Finalmente, la longitud de la muestra (desde 2005 a 2010) permite tener una visión temporal de las decisiones tomadas por las empresas de la muestra, fundamental para un análisis profundo de las mismas.

Al tratarse de una muestra es necesario presentar los datos de la significatividad, cuestión que se discute en el anexo 1. Aquí simplemente indicar que con los habituales criterios de significatividad la muestra de EESs presentan una significatividad un poco por encima del $10 \%$ para cada uno de los años utilizados en el caso de que se considere muestreo aleatorio, pero que esa significatividad es manifiestamente mejor si se interpreta como un muestreo estratificado. Bajo nuestro punto de vista la información disponible justifica la realización de este trabajo.

A pesar de valiosa información que ofrece la ESEE, sólo unos pocos trabajos la han utilizado para estudiar las estrategias y comportamientos de las Empresas de Economía Social. La ESEE distingue entre 1) Empresa Individual, 2) Sociedad Anónima, 3) Sociedad Limitada, 4) Sociedad Anónima Laboral, 5) Cooperativa de Trabajo; y 5) Otras, que será la que se utilice para diferenciar el modelo organizativo en este estudio.

El porcentaje de empresas de la Economía Social (sociedades cooperativas de trabajo y Sociedades Anónimas Laborales) sobre el total de la muestra es del 2,86\%, mostrando un ligero descenso con la crisis. Estos porcentajes suponen una cierta sobrerrepresentación de la Economía Social en la muestra sobre lo que ocurre en la población, donde las empresas de Economía Social representan el 1,8\% de las empresas con 10 o más trabajadores (2.702 sobre un total de 150.064 empresas) $)^{5}$.

Tabla 3.1. Número de empresas por forma jurídica y año

\begin{tabular}{lccccc|cc}
\hline & $\begin{array}{c}\text { Sociedad } \\
\text { Anónima }\end{array}$ & $\begin{array}{c}\text { Sociedad } \\
\text { limitada }\end{array}$ & $\begin{array}{c}\text { Sociedad } \\
\text { anónima laboral }\end{array}$ & $\begin{array}{c}\text { Cooperativa } \\
\text { de trabajo }\end{array}$ & $\begin{array}{c}\text { Otros tipos } \\
\text { de empresa }\end{array}$ & TOTAL & $\begin{array}{c}\text { Porcentaje } \\
\text { Empresa social }\end{array}$ \\
\hline 2005 & 1.093 & 725 & 30 & 28 & 35 & 1.911 & $3,04 \%$ \\
2006 & 1.047 & 879 & 32 & 27 & 38 & 2.023 & $2,92 \%$ \\
2007 & 1.042 & 887 & 27 & 34 & 23 & 2.013 & $3,03 \%$ \\
2008 & 1.054 & 875 & 26 & 32 & 22 & 2.009 & $2,89 \%$ \\
2009 & 1.027 & 921 & 24 & 31 & 12 & 2.015 & $2,73 \%$ \\
2010 & 1.017 & 923 & 20 & 31 & 15 & 2.006 & $2,54 \%$ \\
\hline Total & 6.280 & 5.210 & 159 & 183 & 145 & 11.977 & $2,86 \%$ \\
\hline \multicolumn{7}{c}{ Fuente: Encuesta sobre Estrategias Empresariales }
\end{tabular}

\footnotetext{
${ }^{5}$ Los datos sobre empresas de Economía Social han sido recogidos del Ministerio de Empleo y Seguridad Social, mientras que el número de empresas con 10 o más trabajadores procede del Directorio Central de Empresas (DIRCE).
} 
La distribución por actividades económicas para el año 2010 se presenta en la tabla 3.2, mostrando que las Empresas de Economía Social (EES) se distribuyen de manera heterogénea entre los distintos subsectores de la industria española. Cabe destacar que existen tres sectores, Industria cárnica, productos alimenticios y tabaco, Máquinas agrícolas e industriales y Productos informáticos, electrónicos y ópticos, en los que supera el $4 \%$. En cualquier caso parece que las EESs no surgen en sectores en los que las necesidades de inversión iniciales son elevadas como por ejemplo en Industria química y productos farmacéuticos y Vehículos de motor y otro material de transporte. Sin duda las dificultades del acceso al crédito, así como la presencia de grandes multinacionales en estos sectores son elementos que impiden la implantación de la Economía Social en estos sectores.

Tabla 3.2. Número de empresas por forma jurídica y sector de actividad (Año 2010). Nivel de desagregación 1

\begin{tabular}{|c|c|c|c|c|c|c|c|}
\hline & $\begin{array}{l}\text { Sociedad } \\
\text { Anónima }\end{array}$ & $\begin{array}{l}\text { Sociedad } \\
\text { limitada }\end{array}$ & $\begin{array}{c}\text { Sociedad } \\
\text { anónima } \\
\text { laboral }\end{array}$ & $\begin{array}{c}\text { Cooperativa } \\
\text { de trabajo }\end{array}$ & $\begin{array}{l}\text { Otros } \\
\text { tipos de } \\
\text { empresa }\end{array}$ & TOTAL & $\% \mathrm{ES}$ \\
\hline $\begin{array}{l}\text { Industria cárnica, Productos alimenticios y tabaco, } \\
\text { Bebidas }\end{array}$ & 174 & 142 & 2 & 12 & 6 & 336 & $4,2 \%$ \\
\hline Textiles y confección, Cuero y calzado & 82 & 98 & 1 & 4 & 2 & 187 & $2,7 \%$ \\
\hline $\begin{array}{l}\text { Industria de la madera, Industria del papel, Artes } \\
\text { gráficas }\end{array}$ & 108 & 117 & 3 & 3 & 4 & 235 & $2,6 \%$ \\
\hline Industria química y productos farmacéuticos & 89 & 46 & 0 & 0 & 0 & 135 & $0,0 \%$ \\
\hline $\begin{array}{l}\text { Productos de caucho y plástico, minerales no } \\
\text { metálicos, metales férreos y no férreosy metálicos }\end{array}$ & 290 & 284 & 9 & 2 & 3 & 588 & $1,9 \%$ \\
\hline Máquinas agrícolas e industriales & 61 & 43 & 1 & 4 & 0 & 109 & $4,6 \%$ \\
\hline $\begin{array}{l}\text { Productos informáticos, electrónicos y ópticos, } \\
\text { Maquinaria y material eléctrico }\end{array}$ & 56 & 54 & 2 & 3 & 0 & 115 & $4,3 \%$ \\
\hline Vehículos de motor, Otro material de transporte & 91 & 48 & 0 & 1 & 0 & 140 & $0,7 \%$ \\
\hline $\begin{array}{l}\text { Industria del mueble, Otras industrias } \\
\text { manufactureras }\end{array}$ & 66 & 91 & 2 & 2 & 0 & 161 & $2,5 \%$ \\
\hline Total & 1.017 & 923 & 20 & 31 & 15 & 2.006 & $2,5 \%$ \\
\hline
\end{tabular}

\section{Nivel de desagregación 2}

\begin{tabular}{|c|c|c|c|c|c|c|c|}
\hline \multicolumn{8}{|c|}{ NÚMERO DE EMPRESAS POR SECTOR } \\
\hline ACTIVIDAD & $\begin{array}{l}\text { Sociedad } \\
\text { Anónima }\end{array}$ & $\begin{array}{c}\text { Socieda } \\
\mathrm{d} \\
\text { Limitad } \\
\mathrm{a} \\
\end{array}$ & $\begin{array}{c}\text { Sociedad Anónima } \\
\text { Laboral }\end{array}$ & $\begin{array}{c}\text { Cooperativ } \\
\text { a de } \\
\text { Trabajo }\end{array}$ & Otras & TOTAL & $\begin{array}{c}\text { Peso Economía } \\
\text { Social }\end{array}$ \\
\hline Industria cárnica & 47 & 25 & 0 & 2 & 0 & 74 & $2,70 \%$ \\
\hline Productos alimenticios y tabaco & 101 & 103 & 2 & 5 & 6 & 217 & $3,23 \%$ \\
\hline Bebidas & 26 & 14 & 0 & 5 & 0 & 45 & $11,11 \%$ \\
\hline Textiles y confección & 66 & 62 & 0 & 3 & 2 & 133 & $2,26 \%$ \\
\hline Cuero y calzado & 16 & 36 & 1 & 1 & 0 & 54 & $3,70 \%$ \\
\hline Industria de la madera & 27 & 47 & 2 & 1 & 0 & 77 & $3,90 \%$ \\
\hline Industria del papel & 46 & 33 & 0 & 0 & 0 & 79 & $0,00 \%$ \\
\hline Artes gráficas & 35 & 37 & 1 & 2 & 4 & 79 & $3,80 \%$ \\
\hline $\begin{array}{l}\text { Industria química y productos } \\
\text { farmacéuticos }\end{array}$ & 89 & 46 & 0 & 0 & 0 & 135 & $0,00 \%$ \\
\hline Productos de cauchos y plástico & 56 & 57 & 0 & 0 & 0 & 113 & $0,00 \%$ \\
\hline
\end{tabular}




\begin{tabular}{lccccc|cc} 
Productos minerales no metálicos & 74 & 74 & 1 & 0 & 2 & 151 & $0,66 \%$ \\
\hline Metales férreos y no férreos & 50 & 23 & 1 & 0 & 1 & 75 & $1,33 \%$ \\
\hline Productos metálicos & 110 & 130 & 7 & 2 & 0 & 249 & $3,61 \%$ \\
\hline Máquinas agrícolas e industriales & 61 & 43 & 1 & 4 & 0 & 109 & $4,59 \%$ \\
\hline $\begin{array}{l}\text { Prod. Informáticos, electrónicos y } \\
\text { ópticos }\end{array}$ & 19 & 9 & 1 & 2 & 0 & 31 & $9,68 \%$ \\
\hline Maquinaria y material eléctrico & 37 & 45 & 1 & 1 & 0 & 84 & $2,38 \%$ \\
\hline Vehículos de motor & 63 & 33 & 0 & 1 & 0 & 97 & $1,03 \%$ \\
\hline Otro material de transporte & 28 & 15 & 0 & 0 & 0 & 43 & $0,00 \%$ \\
\hline Industria del mueble & 42 & 62 & 2 & 1 & 0 & 107 & $2,80 \%$ \\
\hline Otras industrias manufactureras & 24 & 29 & 0 & 1 & 0 & 54 & $1,85 \%$ \\
\hline TOTAL & 1.017 & 923 & 20 & 31 & 15 & 2.006 & $2,54 \%$ \\
\hline
\end{tabular}

Fuente: Encuesta sobre Estrategias Empresariales 2010.

Esta distribución es aún más heterogénea si se desagregan aún más los sectores, como aparece en la tabla 3.2 puesto que en este caso aparecen sectores en los que la participación de las EESS supera el 10\% como es el caso de Bebidas.

Para profundizar en el comportamiento relativo a las exportaciones y la innovación de las organizaciones, es útil analizar las tendencias y así poder caracterizar correctamente el comportamiento de los distintos tipos de empresas. Tanto exportaciones como innovación son procesos dinámicos, en los que las decisiones empresariales solo pueden ser vistas como elementos que afectan al futuro de la organización que las toma. En consecuencia, es esperable la presencia de tendencias que la base de datos utilizada permite detectar. A continuación, se estudia la presencia (o no) de estas tendencias con el fin de comprobar si los procesos observados son convergentes o divergentes entre las entidades pertenecientes a la economía social y el resto de empresas industriales en España.

La información disponible permite analizar la evolución tanto en valores absolutos como relativos entendiendo éste como la diferencia entre la empresa de economía social promedio y la correspondiente a las demás formas mercantiles. Los motivos para ofrecer valores relativos es que la información procede de una encuesta, con lo que las cifras absolutas no se corresponden con lo efectivamente realizado en la industria española, mientras que los relativos sí que son significativos puesto que la encuesta es lo suficientemente representativa. ${ }^{6}$ De la misma manera, el análisis se realiza sobre el comportamiento medio, no

\footnotetext{
${ }^{6}$ Así lo demuestra el volumen de trabajos realizados a partir de la ESEE. Por ejemplo, numerosos artículos relacionados con la base de datos y las tesis doctorales realizadas pueden ser encontrados en la web de la Fundación SEPI (http://www.fundacionsepi.es/esee/sp/sesee articulos 1.asp). La discusión acerca de la representatividad de las empresas de economía social se ha planteado en la tabla 3.1 .
} 
pudiendo realizar un análisis regional detenido puesto que al tratarse de una encuesta algunas Comunidades Autónomas no presentan información suficiente para caracterizar el comportamiento de este grupo de organizaciones.

En la Tabla 3.3 se presentan la serie de valores para los años analizados referidos a la situación global de algunas variables relevantes en la actividad de las empresas. La consideración del periodo comprendido entre 2005 y 2010 permite vislumbrar algunos efectos de la crisis económica iniciada en el 2008 y que está teniendo consecuencias ciertamente negativas sobre la economía española en general y el sector industrial, en particular.

El primer elemento de análisis es la evolución en términos de tamaño, entendido como el número de organizaciones. En la Tabla 3.3 se presenta el número de entidades que adoptan la forma jurídica de Sociedades Laborales y Cooperativas, Empresas de la Economía Social (EES), así como el porcentaje que suponen sobre el total de empresas. Destaca el hecho de que la crisis iniciada en el 2008 ha supuesto un descenso continuado en el peso relativo de estas entidades, con lo que el número presenta una clara tendencia regresiva en términos comparativos con respecto al resto del sector industrial, que se mantiene al considerar el valor absoluto.

En términos de valor añadido y ventas destaca el hecho de que la importancia de la economía social era descendente hasta la llegada de la crisis. A partir de este momento cambia el comportamiento si se exceptúa el último de los años considerados. Estos elementos vienen a confirmar la idea de que las empresas de carácter social tienden a mantenerse con mayor frecuencia en momentos de crisis dado su objeto social. Este hecho se corrobora al analizar la evolución del empleo de estas entidades.

Tabla 3.3. Porcentajes de peso de la Economía Social sobre el total del sector industrial en la ESEE.

\begin{tabular}{cccccc}
\hline & Número de empresas & Valor añadido & Ventas & Trabajadores & $\begin{array}{c}\text { Inversión bienes de } \\
\text { equipo }\end{array}$ \\
\hline 2005 & $3,04 \%$ & $1,80 \%$ & $1,33 \%$ & $2,49 \%$ & $1,40 \%$ \\
2006 & $2,92 \%$ & $1,50 \%$ & $0,81 \%$ & $1,91 \%$ & $1,10 \%$ \\
2007 & $3,03 \%$ & $1,91 \%$ & $1,48 \%$ & $2,66 \%$ & $1,43 \%$ \\
2008 & $2,89 \%$ & $2,09 \%$ & $1,49 \%$ & $2,51 \%$ & $1,37 \%$ \\
2009 & $2,73 \%$ & $2,50 \%$ & $1,78 \%$ & $2,65 \%$ & $1,17 \%$ \\
2010 & $2,54 \%$ & $1,59 \%$ & $1,53 \%$ & $2,28 \%$ & $1,33 \%$ \\
\hline
\end{tabular}


La evolución de las cifras anteriores alerta acerca de la brecha en productividad de los dos modelos de empresas consideradas. El hecho de que la importancia relativa en número de trabajadores sea claramente superior, con excepción del 2009, a la cifra asociada a ventas y a valor añadido indica que las entidades de economía social son menos productivas en términos de producción por trabajador. Este hecho puede deberse a los sectores de actividad en los que se localizan o a los datos que ofrece la última de las columnas de la Tabla 3.3 que se refieren a la inversión en bienes de equipo. En este caso se observa que las organizaciones de economía social presentan un porcentaje de inversión en bienes de equipo claramente inferior al de resto de entidades industriales, incluso el porcentaje desciende a partir de 2008, inicio de la crisis, dando razones para entender la evolución de la productividad.

Las cifras anteriores pueden estar sesgadas dependiendo del tamaño de las entidades. Por este motivo se ha considerado la empresa media de cada tipo de sociedad, presentando en la Tabla 3.4 la diferencia entre las de Economía Social (EES) y el resto de entidades en términos porcentuales. En primer lugar, cabe señalar que la Empresa de Economía Social (EES) media es de menor tamaño en términos de trabajadores que la correspondiente al resto de entidades, aunque parece cierto grado de convergencia. Este resultado se deducía, no obstante, de la comparación del porcentaje de empresas y de trabajadores de la Tabla 3.3.

Tabla 3.4. Comparación entre la empresa media de Economía Social y la media del resto de sociedades.

\begin{tabular}{ccccc}
\hline & Valor añadido & Ventas & Trabajadores & $\begin{array}{c}\text { Inversión bienes de } \\
\text { equipo }\end{array}$ \\
\hline 2005 & $58,14 \%$ & $43,01 \%$ & $81,53 \%$ & $45,26 \%$ \\
2006 & $50,66 \%$ & $27,06 \%$ & $64,94 \%$ & $36,95 \%$ \\
2007 & $62,22 \%$ & $47,98 \%$ & $87,34 \%$ & $46,37 \%$ \\
2008 & $71,34 \%$ & $50,86 \%$ & $86,60 \%$ & $46,74 \%$ \\
2009 & $90,84 \%$ & $64,66 \%$ & $97,01 \%$ & $42,04 \%$ \\
2010 & $61,97 \%$ & $59,42 \%$ & $89,57 \%$ & $51,88 \%$ \\
\hline
\end{tabular}

Llama la atención la evolución de los porcentajes del valor añadido y de ventas, que antes de la crisis presentaban una tendencia decreciente y que cambia de sentido al comenzar la misma. En todo caso, los valores de las EES promedio se sitúan por debajo de los valores del resto de empresas (ninguno supera el 100\%, que supondrían que las EES están por encima). Esto ocurre especialmente en lo referido a ventas. Ese cambio de tendencia es todavía más claro si atendemos a las cifras correspondientes a la inversión en bienes de equipo, la única en la que estas organizaciones se acercan al resto en el 2010. 
En conjunto, la crisis parece haber abierto un proceso de convergencia en términos de los distintos valores generales analizados que se "rompe" en el 2010, quizá por la duración de este periodo de crisis, que acaba afectando a todos los modelos de empresa. Se hace necesario disponer de información sobre años posteriores para comprobar si se trata de un hecho puntual o un cambio de tendencia.

Una vez analizado el comportamiento agregado, resulta interesante centrase en los posibles cambios en la evolución que aparecen en términos de comportamiento estratégico relacionado con las dos variables centrales de este informe, las exportaciones y las actividades de I+D. Sin duda el contexto de crisis actual está suponiendo un replanteamiento de las estrategias de las organizaciones. Un primer aspecto es la importante contracción de la demanda interna o nacional que hace obligatorio una apertura de las empresas a los mercados internacionales con el objeto de mantener sus ventas. En la Tabla 3.5 aparecen los porcentajes de empresas exportadoras para las dos agrupaciones consideradas: las asociadas a la economía social y el resto. En la evolución observada se comprueba de manera evidente el esfuerzo de Sociedades Laborales y Cooperativas, tendente a ampliar sus mercados fuera del espacio nacional. De hecho, al principio de la muestra alrededor del $40 \%$ atendían a la demanda extranjera mientras que al final se llega a superar el 60\%, es decir se produce un aumento de casi 20 puntos porcentuales que supera de manera clara lo acontecido en el resto de empresas. Sin embargo, el porcentaje de las EES se sitúa en todos los años por debajo del que presentan el resto de entidades mercantiles, debiendo intensificar el esfuerzo en esta dirección, cuestión que se viene dando en los últimos ocho años que son para los que se dispone de información. Algo similar sucede al considerar las actividades de I+D.

Tabla 3.5. Porcentaje de empresas exportadoras y que realizan actividades de I+D.

\begin{tabular}{c|ccc|ccc}
\hline & \multicolumn{3}{|c|}{ Empresas exportadoras } & \multicolumn{3}{c}{ Empresas con actividades de I+D } \\
\hline & Otras & Economía Social & Brecha & Otras & Economía Social & Brecha \\
\hline 2005 & $63,26 \%$ & $41,38 \%$ & 21,88 & $37,94 \%$ & $27,59 \%$ & 10,35 \\
2006 & $62,56 \%$ & $54,10 \%$ & 8,46 & $35,39 \%$ & $30,51 \%$ & 4,88 \\
2007 & $63,98 \%$ & $56,90 \%$ & 7,08 & $34,37 \%$ & $32,79 \%$ & 1,58 \\
2008 & $63,98 \%$ & $56,90 \%$ & 7,08 & $35,37 \%$ & $36,21 \%$ & $-0,84$ \\
2009 & $64,85 \%$ & $52,73 \%$ & 12,12 & $34,74 \%$ & $38,18 \%$ & $-3,44$ \\
2010 & $65,80 \%$ & $60,78 \%$ & 5,02 & $35,55 \%$ & $31,37 \%$ & 4,18 \\
\hline
\end{tabular}


Los cuadros anteriores permiten establecer el marco general en el que analizar las diferencias en el comportamiento en términos de exportaciones y actividad innovadoras de ambos tipos de entidades, cuestión que abordamos en las dos secciones siguientes.

\section{ACTIVIDAD EXPORTADORA}

Además de las cifras en términos de empresas, resulta interesante analizar la evolución de las cifras totales, tanto en términos del grado de apertura al sector exterior. Con respecto al primero de estos aspectos, la Tabla 4.1 ofrece algunos indicadores. En términos de mercados exteriores, las EES están ganando peso en ventas, dato que se intensifica si se considera la empresa promedio. La crisis está llevando a las EES a un contexto en el que la internacionalización se convierte en un elemento estratégico muy relevante. Es posible que este proceso esté "dañando" la vertiente localista de estas entidades, cuestión que es difícil de analizar. En todo caso, esta creciente apertura aparece, sobre todo, si se analizan las importaciones, puesto que en este aspecto el valor máximo aparece al final de la muestra, lo que podría indicar la apertura de estas empresas buscando productos en el mercado exterior.

Tabla 4.1. Porcentaje de exportaciones e importaciones sobre el total y comparación de medias entre empresas sociales y el resto

\begin{tabular}{c|cc|cc}
\hline & \multicolumn{2}{|c|}{ Exportaciones } & \multicolumn{2}{c}{ Importaciones } \\
\hline & Economía Social & $\begin{array}{c}\text { Comparación con el } \\
\text { resto de sector }\end{array}$ & Economía Social & $\begin{array}{c}\text { Comparación con el } \\
\text { resto de sector }\end{array}$ \\
\hline 2005 & $1,43 \%$ & $45,99 \%$ & $0,91 \%$ & $29,30 \%$ \\
2006 & $1,01 \%$ & $33,95 \%$ & $0,17 \%$ & $5,72 \%$ \\
2007 & $1,37 \%$ & $44,55 \%$ & $1,06 \%$ & $34,41 \%$ \\
2008 & $1,35 \%$ & $45,96 \%$ & $0,78 \%$ & $26,33 \%$ \\
2009 & $1,68 \%$ & $60,83 \%$ & $0,75 \%$ & $26,97 \%$ \\
2010 & $1,58 \%$ & $61,41 \%$ & $0,96 \%$ & $37,02 \%$ \\
\hline
\end{tabular}

En términos comparativos entre empresas de los dos grupos, parece claro como la EES media está centrando sus esfuerzos en el mercado exterior, al pasar de suponer el $46 \%$ de las ventas del resto de empresas en 2005 a superar el 60\% de las ventas en 2010. Estas cifras son claramente indicativas de un cambio estratégico, al menos en promedio, con una transformación importante en los mercados a los que las EES dirigen su actividad productiva. Es reseñable que estos indicios de apertura internacional se reflejan también en las cifras de importaciones. En este caso, es más difícil de interpretar, puesto que la EES se localiza en un espacio geográfico concreto que determina sus proveedores en gran medida. A pesar de esto, 
parece que están optando por cubrir sus necesidades con inputs del mercado exterior, lo que puede suponer un abaratamiento de costes de producción.

Asimismo, un elemento importante es la distribución de las exportaciones. La información disponible no es anual en este caso, pero de igual manera permite conocer los mercados geográficos a los que se dirigen las ventas de las distintas empresas. La apertura de nuevos mercados tiene que ver en gran medida con el acceso a ellos, y en la Tabla 4.2 se muestra la distribución de las exportaciones. Destaca que la dicha distribución apenas se ha modificado con la crisis en el caso de las EESs mientras que el resto muestra una importancia mayor de las ventas al exterior de la CEE.

Tabla 4.2. Distribución de las exportaciones según destino.

\begin{tabular}{llcc}
\hline \multirow{3}{*}{ Empresas sociales } & & 2006 & 2010 \\
& CEE & $74,20 \%$ & $74,76 \%$ \\
& Iberoamérica & $5,11 \%$ & $3,72 \%$ \\
\multirow{3}{*}{ Otras } & Resto & $20,69 \%$ & $21,52 \%$ \\
\hline & CEE & $73,56 \%$ & $69,19 \%$ \\
& Iberoamérica & $5,03 \%$ & $4,29 \%$ \\
& Resto & $21,42 \%$ & $26,52 \%$ \\
\hline
\end{tabular}

CEE: Comunidad Económica Europea

OCDE: Organización para la Cooperación y el Desarrollo Económicos

Obviamente, también es importante conocer el comportamiento de las empresas en términos de mercado interior. En la Tabla 4.3 se muestra la distribución de las empresas que declaran su mercado como local, provincial, regional y nacional en el caso de las EES y del resto. Lo primero que destaca en que la variabilidad es mucho más intensa para las primeras, al no ser posible establecer ningún tipo de regularidad. Este comportamiento cambia en el mercado regional, que es creciente durante la expansión económica y se contraerse a partir de 2008. En el resto del sector industrial se observa una caída del mercado provincial, e incluso del nacional, a favor del mercado exterior, como se ha comentado con anterioridad.

Tabla 4.3. Distribución del mercado interior

\begin{tabular}{ccccc}
\hline \multicolumn{5}{c}{ Empresas de Economía Social } \\
\hline 2005 & Local & Provincial & Regional & Nacional \\
\hline 2006 & $8,62 \%$ & $10,34 \%$ & $13,79 \%$ & $39,66 \%$ \\
2007 & $11,86 \%$ & $13,56 \%$ & $20,34 \%$ & $30,51 \%$ \\
2008 & $8,20 \%$ & $8,20 \%$ & $11,48 \%$ & $40,98 \%$ \\
2009 & $6,90 \%$ & $6,90 \%$ & $10,34 \%$ & $43,10 \%$
\end{tabular}




\begin{tabular}{ccccc}
2010 & $11,76 \%$ & $7,84 \%$ & $9,80 \%$ & $41,18 \%$ \\
\hline \multicolumn{5}{c}{ Resto de Empresas } \\
\hline 2005 & Local & Provincial & Regional & Nacional \\
2006 & $6,48 \%$ & $11,28 \%$ & $8,69 \%$ & $41,99 \%$ \\
2007 & $6,25 \%$ & $10,44 \%$ & $10,18 \%$ & $42,11 \%$ \\
2008 & $6,66 \%$ & $9,94 \%$ & $10,4 \%$ & $43,14 \%$ \\
2009 & $6,17 \%$ & $8,82 \%$ & $10,87 \%$ & $40,85 \%$ \\
2010 & $6,39 \%$ & $8,47 \%$ & $10,66 \%$ & $41,17 \%$ \\
\hline
\end{tabular}

\section{ACTIVIDAD INNOVADORA}

La actividad en innovación también presenta elementos que deben ser considerados a nivel descriptivo. En la Tabla 5.1 aparecen algunos de estos elementos. En primer lugar, destaca que la crisis ha supuesto que los montantes destinados a I+D en la Economía Social se han visto claramente aumentados, con lo que parece evidente que aquellas empresas que no dedicaban recursos a estos aspectos están siendo expulsadas del mercado. Este resultado se refuerza cuando se compara la empresa media de ambos sectores, puesto que se observa que es uno de los indicadores en los que ambos tipos de entidades se encuentran más cercanas.

El método de financiación de las actividades de I+D es también importante, y más si se tiene en cuenta que las políticas de carácter autonómico están marcando, en gran medida, este tipo de actividades. En este caso, la crisis ha supuesto un acercamiento de las EES al acceso a estos fondos que les corresponden, si se atiende a su importancia en términos de empleo o ventas. La consideración del sector público como ente financiador de estas actividades debe ser una variable relevante desde este punto de vista. El gobierno desde sus distintos departamentos o agencias contribuye a este tipo de acción. Entre las actuaciones relevantes aparecen las de formación, ya que es un elemento clave a la hora de implementar las mejoras asociadas a las actividades de I+D.

En la última columna de la Tabla 5.1 se muestra el porcentaje de los gastos en formación que realizan las EES de la muestra. Como puede comprobarse, estos gastos no se corresponden con la evolución de otras variables analizadas sino que se sitúa claramente por debajo de la importancia de estas entidades en términos de producción o ventas. En resumen, estas cifras muestran que la formación es una clara área de mejora en términos estratégicos, puesto que la implementación de mejoras técnicas no puede disociarse de una formación de 
los trabajadores que son, en definitiva, los que las llevan a cabo de manera efectiva. Algo similar resulta a la hora de comparar los gastos en $\mathrm{I}+\mathrm{D}$, aunque en este caso al comparar las empresas medias el resultado indica que se han aproximado bastante al final de la muestra.

Tabla 5.1. Indicadores de innovación y formación.

\begin{tabular}{ccccc}
\hline & $\begin{array}{c}\text { Gastos I+D Economía } \\
\text { Social }\end{array}$ & $\begin{array}{c}\text { Comparación con el resto de } \\
\text { sector }\end{array}$ & $\begin{array}{c}\text { Gastos I+D con financiación } \\
\text { pública en EES }\end{array}$ & $\begin{array}{c}\text { Gastos formación } \\
\text { Economía Social }\end{array}$ \\
\hline 2005 & $1,97 \%$ & $64,10 \%$ & $1,79 \%$ & $2,17 \%$ \\
2006 & $0,87 \%$ & $28,99 \%$ & $2,03 \%$ & $1,82 \%$ \\
2007 & $1,99 \%$ & $64,87 \%$ & $3,50 \%$ & $1,24 \%$ \\
2008 & $2,63 \%$ & $90,53 \%$ & $2,85 \%$ & $1,47 \%$ \\
2009 & $2,55 \%$ & $93,33 \%$ & $2,01 \%$ & $1,58 \%$ \\
2010 & $2,26 \%$ & $88,83 \%$ & $1,85 \%$ & - \\
\hline
\end{tabular}

Otro elemento a considerar dentro de la política estratégica es la organización de las actividades de I+D. En términos de gestión, la cuestión tiene que ver con la decisión de desarrollar o no un departamento interno que se centre en este tipo de actividades, o externalizar estas actividades, contratando empresas dedicadas a ellas, o una combinación de ambas. Sin duda el desarrollo de un departamento de innovación dentro de las entidades permite una mayor dedicación a esta actividad, aunque también supone un mayor riesgo puesto que la no consecución de resultados incrementa los costes de las entidades de manera notable.

En los datos que aparecen en la Tabla 5.2 se muestra claramente que el perfil dinámico del comportamiento de las EES difiere notablemente del seguido por el resto de empresas. En este caso, destaca que mientras en las segundas la combinación de innovación interna y externalizada gana peso, en las primeras ocurre lo contrario. Esto quiere decir que en la Economía Social se está optando por una especificación de las actividades de I+D, de manera que se está eliminando el modelo que podríamos denominar como mixto, al contrario que el resto de organizaciones. El análisis acerca de la financiación de este tipo de actividades no es sencillo, puesto que el hecho de que no resulte claro el retorno de la actividad, ni siquiera su tasa de éxito, hace que en muchos casos los gerentes de las empresas tengan objeciones a la hora de ponerlas en marcha. 
Tabla 5.2. Porcentaje de empresas con $I+D$ según el origen de la innovación.

\begin{tabular}{l|cc|cc|cc}
\hline & \multicolumn{2}{|c|}{ Innovación interna } & \multicolumn{2}{c|}{ Innovación externa } & \multicolumn{2}{c}{ Ambas } \\
\hline & Otras & Economía Social & Otras & Economía Social & Otras & Economía Social \\
\hline 2005 & 13,6 & 1,72 & 6,58 & 3,45 & 17,75 & 22,41 \\
2006 & 12,12 & 8,47 & 5,5 & 1,69 & 17,77 & 20,34 \\
2007 & 11,63 & 11,48 & 5,43 & 3,28 & 17,32 & 18,03 \\
2008 & 12,25 & 10,34 & 5,69 & 6,90 & 17,43 & 18,97 \\
2009 & 11,22 & 12,73 & 5,05 & 7,27 & 18,47 & 18,18 \\
2010 & 12,07 & 7,84 & 4,35 & 5,88 & 19,13 & 17,65 \\
\hline
\end{tabular}

Hasta ahora se ha analizado la intensidad en actividades de $\mathrm{I}+\mathrm{D}$ en términos de número de empresas. Más interesante resulta utilizar otro tipo de indicadores asociados al gasto realizado o a la innovación de productos y/o procesos. En cuanto al gasto por empresa, la Tabla 5.3 ofrece resultados interesantes. En este caso, se observa como la brecha se reduce desde el 35\% en 2005 a poco más del 10, y resulta importante señalar que la crisis ha detenido la tendencia creciente de este tipo de gasto en el resto de empresas pero no parece haber tenido el mismo efecto en la EESs. En este segundo caso, las cantidades, exceptuando el año 2006, siempre presenta un crecimiento sostenido, que supera el 23\% en el 2008. Por tanto, nos encontramos con modelo organizativo (el de las EES) que pierde importancia en término de "población", pero que gana peso en el esfuerzo monetario en actividades I+D. De hecho, la brecha en términos porcentuales se sitúa por debajo del $7 \%$ en 2009 , muy lejos del 35,9\% observado a principios en 2005 , lo que da una idea del cambio en términos estratégicos de estas entidades.

Tabla 5.3. Gasto medio en I+D por tipo de empresa.

\begin{tabular}{lccc}
\hline & Economía Social & Otras & Diferencia \\
\hline 2005 & $682.678,1$ & $1.065 .037,0$ & $-35,90 \%$ \\
2006 & $340.377,8$ & $1.173 .957,0$ & $-71,01 \%$ \\
2007 & $747.028,5$ & $1.151 .617,0$ & $-35,13 \%$ \\
2008 & $919.501,1$ & $1.015 .635,0$ & $-9,47 \%$ \\
2009 & $1.011 .253,0$ & $1.083 .492,0$ & $-6,67 \%$ \\
2010 & $942.106,70$ & $1.060 .581,00$ & $-11,17 \%$ \\
\hline
\end{tabular}

Sin embargo, el gasto en I+D y la efectividad que éste tiene no siempre van en consonancia. En este caso, los datos disponibles ofrecen información acerca del número de innovaciones de producto y de proceso que han tenido lugar en las empresas encuestadas en términos de número de resultados medios. Éstos se presentan en la Tabla 5.4, pudiendo establecer la efectividad de los gastos realizados. 
Tabla 5.4. Innovación de producto y de proceso por empresa.

\begin{tabular}{l|ccc|ccc}
\hline & \multicolumn{3}{|c|}{ Innovación de producto } & \multicolumn{3}{c}{ Innovación de proceso } \\
\hline & Economía Social & Resto del sector & Dif. & Economía Social & Resto del sector & Dif. \\
\hline 2005 & 0,138 & 0,223 & $-0,085$ & 0,138 & 0,290 & $-0,152$ \\
2006 & 0,136 & 0,207 & $-0,071$ & 0,220 & 0,280 & $-0,059$ \\
2007 & 0,213 & 0,189 & 0,025 & 0,410 & 0,351 & 0,059 \\
2008 & 0,172 & 0,180 & $-0,008$ & 0,345 & 0,341 & 0,004 \\
2009 & 0,127 & 0,193 & $-0,066$ & 0,273 & 0,328 & $-0,055$ \\
2010 & 0,176 & 0,203 & $-0,026$ & 0,314 & 0,346 & $-0,033$ \\
\hline
\end{tabular}

La Tabla 5.4 muestra que el comportamiento de cada modelo de empresa varía dependiendo de si nos referimos a innovación de producto o de proceso. En el primero tipo de innovación, la diferencia de resultados se mantiene en el periodo considerado a favor de las empresas que no se clasifican como Economía Social. En las innovaciones de proceso, la brecha inicial es mucho mayor, pero se ha reducido considerablemente y en algunos periodos (principalmente en los años 2007 y 2008) ha llegado a ser a favor de las EES. Por tanto, parece claro que el incremento de los montantes destinados a los procesos innovadores de las EES se ha dirigido a la innovación de procesos y no tanto a la de producto.

Finalmente, a continuación se presentan dos tipos de datos adicionales, que se refieren a las patentes conseguidas por los distintos tipos de entidades. Las patentes permiten la disposición en régimen de monopolio temporal de la utilización de un adelanto técnico, tanto en términos de producto como de proceso, pudiendo utilizarse como indicador de los resultados de la política de I+D. En la Tabla 5.5 destaca la capacidad de las EES para generar patentes, puesto que es de los pocos indicadores en los que se sitúa por encima del resto del sector industrial, y además de manera clara. Merecen especial atención las patentes inscritas fuera de España, puesto que en los últimos años las EES han mostrado un comportamiento más incisivo en este sentido.

Tabla 5.5. Patentes de las empresas industriales españolas.

\begin{tabular}{l|cc|cc}
\hline & \multicolumn{2}{|c|}{ Economía Social } & \multicolumn{2}{c}{ Resto del Sector } \\
\hline & Nacionales & Extranjeras & Nacionales & Extranjeras \\
\hline 2005 & 0,21 & 0,36 & 0,19 & 0,24 \\
2006 & 0,49 & 0,49 & 0,15 & 0,30 \\
2007 & 0,16 & 0,31 & 0,15 & 0,36 \\
2008 & 0,24 & 0,52 & 0,15 & 0,32 \\
2009 & 0,31 & 0,20 & 0,16 & 0,35 \\
2010 & 0,57 & 0,65 & 0,31 & 0,19 \\
\hline
\end{tabular}


En resumen, en este apartado se ha presentado la evolución dinámica de las empresas industriales españolas desde una doble perspectiva: Por un lado, desde una perspectiva dinámica, con el fin de comprobar el efecto de la crisis que comenzó en 2008 y que ha supuesto un cambio considerable en estrategias y políticas comerciales. Por otro, desde la visión que supone segmentar las organizaciones entre Empresas de Economía Social (Cooperativas de Trabajo Asociado y Sociedades Laborales) y las del resto del sector. Las tablas presentadas muestran comportamientos distintos entre los dos modelos organizativos en ambas perspectivas.

\section{CONCLUSIONES}

La actual crisis ha puesto de manifiesto la necesidad de analizar el comportamiento estratégico de las empresas tradicionales y de las cooperativas con el objetivo de saber cuáles de estas estrategias contribuyen a la continuidad de estas entidades. En concreto, la innovación y la intensidad exportadora aparecen de manera recurrente como don elementos muy importantes. En este trabajo se ha realizado una primera aproximación al comportamiento en estas dos dimensiones de la dirección estratégica en las cooperativas y las sociedades anónimas laborales, las denominadas empresas de economía social (EES), del sector industrial español, comparando con las cifras del resto de empresas.

En términos de representatividad, la Encuesta sobre Estrategias Empresariales (ESEE) para empresas industriales españolas mantiene la proporción de EES sobre el total de empresas observada a nivel agregado. En este sentido, una primera aproximación destacar la importancia de las cooperativas en sectores como el de bebidas (debido a las cooperativas vitivinícolas) y el de Productos informáticos, electrónicos y ópticos. También en sectores como la Industria cárnica, Productos alimenticios y tabaco, Textiles y confección, Cuero y calzado, Artes gráficas y Maquinaria agrícola e industrial presentan un valor significativo. En cuanto a las Sociedades Anónimas Laborales suelen estar presentes en los mismos sectores y además en la Industria de la madera, Metales férreos y no férreos y Productos metálicos. Por tanto, se puede aceptar que existe cierta coincidencia en los sectores en los que se ubican ambos tipos de organización.

Si se atiende al tamaño la distribución anterior, el resultado cambia notablemente, puesto que si se consideran las empresas con más de 200 trabajadores la presencia de 
cooperativas en la Industria cárnica es del 7,1\%, en Máquinas agrícolas e industriales el 8,3\% y en Productos informáticos, electrónicos y ópticos el 14,3\%.

Dado el objetivo del artículo, que es describir en términos comparados la evolución de las EES en términos de exportaciones e innovación respecto del resto de empresas y la influencia de la crisis, son varias las conclusiones extraídas del análisis descriptivo.

En términos de valor añadido y ventas destaca el hecho de que la importancia de la economía social era descendente hasta la llegada de la crisis. A partir de este momento cambia el comportamiento si se exceptúa el último de los años considerados.

En términos de mercados exteriores, las EES están ganando peso en ventas puesto que pasan de un $48 \%$ de las ventas al exterior del resto de empresas en 2003 a superar el $60 \%$ de las ventas en 2010. Estas cifras son claramente indicativas de un cambio estratégico, al menos en promedio, con una transformación importante en los mercados a los que las EES dirigen su actividad productiva. Estos indicios de apertura internacional se reflejan también en las cifras de importaciones. La apertura de nuevos mercados por parte de las EES se centra en el aumento exportador a la Unión Europea, que ha pasado del 33\% a casi el $47 \%$.

Por otro lado la crisis ha supuesto que los montantes destinados a $\mathrm{I}+\mathrm{D}$, en términos de gasto sobre el total de gastos de las empresas, en las EES han aumentado claramente, con lo que se ha aproximado en media a los datos observados en el resto de empresas, pasando del 49\% en 2003 AL 88\% en 2010.

En términos de resultados de la innovación, se comprueba que para la innovación de producto la diferencia de resultados se mantiene en el periodo considerado a favor de las empresas que no se clasifican como Economía Socia, aunque la brecha se reduce, principalmente debido a los resultados de los años 2007 y 2008. Por tanto, parece claro que el incremento de los montantes destinados a los procesos innovadores de las EES se ha dirigido a la innovación de procesos y no tanto a la de producto.

Como último indicador de innovación se utilizan las patentes registradas. destaca la capacidad de las EES para generar patentes, puesto que es de los pocos indicadores en los que se sitúa por encima del resto del sector industrial, y además de manera clara. Merecen especial atención las patentes inscritas fuera de España, puesto que en los últimos años las EES han mostrado un comportamiento más incisivo en este sentido. 


\section{BIBLIOGRAFÍA}

ÁGUILA, A. R. y PADILLA, A. (2010) Factores determinantes de la innovación en empresas de Economía Social. La importancia de la formación y de la actitud estratégica, Ciriec España. Revista de Economía Pública Social y Cooperativa, 67, 129-155.

AKYOL, A. y AKEHURST, G. (2003) An investigation of export performance variation related to corporate export market orientation, European Business Review, 13 (1), pp. 23 19

AMAT, O. y PERRAMON, J. (2011) High growth cooperatives: financial profile and key factors for competitiveness, Ciriec España. Revista de Economía Pública Social y Cooperativa, 73, pp. 81-98.

ANTONIOLOLI, D., MAZZANTI, M. y PINI, P. (2011) Innovation, industrial relations and employee outcomes: evidence from Italy, Journal of Economics Studies, 38, pp. 66-90.

BALEREN, B., BEGIRISTAIN, A., ERRASTI, A. y GOIKOETXEA, G. (2004) Redes e innovación cooperativa, Ciriec España. Revista de Economía Pública Social y Cooperativa, 49, pp. 263-294.

CHESBROUGH, H. (2004) Managing Open Innovation Research Technology Management, $\mathrm{Jan} / \mathrm{Fec}, 47$ (1).

CHESBROUGH, H. (2005) Open Innovation, Harvard Business School Press, Boston.

CLEMENTE, J.; DÍAZ, M. y MARCUELLO, C. (2009) Cooperativas y Sociedades Laborales en España: estudio de su contribución al empleo y al crecimiento económico. Revesco. Revista de Estudios Cooperativos, $\mathrm{n}^{\circ} 98$.

ClEMENTE, J.; DÍAZ, M.; MARCUELlO, C. y SANSO, M. (2012) The Wage Gap between Cooperative and Capitalist Firms: Evidence from Spain, Annals of Public and Cooperative Economics, ${ }^{\circ} 83$.

COQUE, J. (2002) La innovación tecnológica en las sociedades cooperativas y otras organizaciones participación, Revesco. Revista de Estudios Cooperativos nº. 78, pp. 9-25.

COQUE, J. y PÉREZ, E. (1999) La actitud ante la innovación tecnológica de pequeñas cooperativas industriales. Una experiencia de promoción del cooperativismo de trabajo asociado mediante investigación-acción participativa. En: Ayala, J.C. (1999): La gestión de la diversidad: Actas del XIII Congreso Nacional y del IX Congreso Hispano-Francés, Vol. 1, pp. 1355-1364. 
DAMANPOUR, F. y GOPALAKRISHNAN, S. (2001) The dynamics of the adoption of product and process innovations in organizations, Journal of Management Studies, Vol. 38 $\mathrm{n}^{\circ} .1, \mathrm{pp} .45-65$.

ECHEVARRÍA, J. (2008) El Manual de Oslo y la Innovación social, Arbor. Ciencia, Pensamiento y Cultura, 732, 609-618.

FERNÁNDEZ, M. V., PEÑA, I. y HERNÁNDEZ, F. (2008) Factores determinantes del éxito exportador. El papel de la estrategia exportadora en las cooperativas agrarias. Ciriec España. Revista de Economía Pública Social y Cooperativa, 63, 39-64.

FLOR, M.L. CAMISÓN, C. y OLTRÁ, M. J. (2004) El efecto de la estrategia de innovación tecnológica y de la estrategia exportadora en el desempeño internacional de la empresa, Cuadernos de Economía y Dirección de la Empresa, 20, pp. 151-174.

FUENTES, F.J., SÁNCHEZ, S.M. y SANTOS, L.M. (2011) Cooperativas agroalimentarias y exportación. El proceso de internacionalización de la Cooperativa del Valle de Los Pedroches (COVAP), Revesco. Revista de Estudios Cooperativos, 104, pp. 38-62.

FUNDACIÓN SEPI (2012) Las Empresas Industriales en 2010, http://www.fundacionsepi.es/esee/sp/sesee_informes.asp

KALMI, P. y KAUHANENE, A. (2008) Workplace innovation and employee outcome evidence from Finland, Industrial Relations, 47 (3), pp. 430-459.

KOSCHATZKY, K. (2002) Fundamentos de la economía de redes. Especial enfoque a la innovación, Economía Industrial, n 348, pp. 15-126.

MORALES GUTIÉRREZ, A. C. (2012) Innovación social y cooperativas: convergencias y sinergias, Ekonomiaz, 79, pp. 146-167.

NAVARRO, A. (2002) Efecto de la estrategia de expansión exterior sobre el resultado exportador de la organización, Revista del Ministerio de Industria, Turismo y Comercio, 802, pp. 99-115. 


\section{ANEXO 1: Grado de significatividad de la muestra, utilizada en el trabajo, en lo referido a las empresas EES.}

Las empresas que participan en la ESEE se seleccionan combinando criterios de exhaustividad y muestreo aleatorio dependiendo del nivel de empleo. A las empresas de más de 200 trabajadores se les requiere exhaustivamente su participación. Sin embargo, las empresas con empleo comprendido entre 10 y 200 trabajadores son seleccionadas mediante muestreo estratificado. Los estratos definidos para el muestreo resultan del cruce de los intervalos de empleo de 10-20, 21-50, 51-100 y 101-200 trabajadores y la actividad económica de las empresas según su CNAE, esto asegura que la encuesta es representativa del sector manufacturero en su conjunto y del tamaño y sector de actividad de las empresas en particular.

Un correcto muestreo estratificado asegura la obtención de un tamaño adecuado para cada uno de los estratos, aunque estos subgrupos sean pequeños, y permite la comparación entre los grupos. Aplicando el procedimiento descrito por Kish $(1965)^{7}$, recogido en el libro de Sampieri, Fernández y Baptista (2006) "Metodologías de la investigación", , se puede determinar si el tamaño del subgrupo de empresas de economía social es suficiente para permitir la comparación entre éstas y el resto de empresas de la muestra.

Para el año 2010 la población de empresas industriales $(\mathrm{N})$ es de 150.064 , el tamaño muestral (n) para ese año es de 2.006 empresas. De acuerdo al procedimiento de estratificación el tamaño mínimo que debe tener el subgrupo de empresas de economía social es el resultante de multiplicar el tamaño poblacional real de empresas de ES (2.702 empresas según datos del Ministerio de Empleo y Seguridad Social) por la fracción resultante de n/N (0.0134), por tanto 37 empresas. Un tamaño de 51 para este año nos permite confiar en la validez de las conclusiones.

Dado que en los años 2003 y 2004 la muestra de empresas de economía social no llega a 50, se ha optado por eliminar esos dos años del análisis, sin embargo, las conclusiones para estos dos años no eran diferentes del resto, por lo que a pesar del menor número de observaciones los resultados son consistentes.

\footnotetext{
${ }^{7}$ KISH, LESLIE. (1965). Survey Sampling. Nueva York: John Wiley \& Sons.

${ }^{8}$ Sampieri, Fernández y Baptista,. 2006. Capítulo 8 de "Metodología de la investigación", McGraw-Hill.
} 
Aunque consideramos que la correcta estratificación de la muestra es suficiente para asegurar la validez de la comparación entre las entidades mercantiles y las empresas de economía social, se ha calculado también el error muestral para los dos grupos de empresas como si éstas fueran muestras independientes. Dado el menor tamaño, el margen de error en las submuestras será siempre mayor que el de la muestra total.

La tabla a.1 presenta el error muestral para un intervalo de confianza del $90 \%$, calculado de acuerdo a la fórmula:

$$
\mathrm{n}=\frac{\mathrm{N} * \mathrm{Z}_{\alpha / 2}^{2} * \mathrm{P} *(1-\mathrm{P})}{(\mathrm{N}-1) * \mathrm{e}^{2}+\mathrm{Z}_{\alpha / 2}^{2} * \mathrm{P} *(1-\mathrm{P})}
$$

donde $e$ es el error muestral o error de estimación a causa de observar una muestra en lugar de la población completa. $Z_{\alpha / 2}$ correspondiente al $Z$ con el nivel de confianza elegido, en este caso el $90 \%$. $P$ es la proporción de una categoría de la variable, se ha optado por calcular el error en la opción más conservadora asignando a $P$ el valor 0,5 . $N$ es el tamaño de la población y $n$ el tamaño de la muestra.

Tabla a.1: Errores muestrales por año: $\mathrm{p}=\mathrm{q}=0,5$ (opción conservadora)

\begin{tabular}{|c|c|c|c|c|c|c|}
\hline & \multicolumn{2}{|c|}{ Muestra completa } & \multicolumn{2}{|c|}{ Resto de empresas } & \multicolumn{2}{|c|}{ Empresas de ES } \\
\hline & $\begin{array}{c}N^{o} \text { de } \\
\text { empresas }\end{array}$ & $\begin{array}{l}\text { Nivel de } \\
\text { error }\end{array}$ & $\begin{array}{c}N^{0} \text { de } \\
\text { emopresas }\end{array}$ & $\begin{array}{c}\text { Nivel de } \\
\text { error }\end{array}$ & $\begin{array}{c}N^{\circ} \text { de } \\
\text { empresas }\end{array}$ & $\begin{array}{c}\text { Nivel de } \\
\text { error }\end{array}$ \\
\hline 2003 & 1.380 & $2,14 \%$ & 1.335 & $2,17 \%$ & 45 & $12,16 \%$ \\
\hline 2004 & 1.374 & $2,14 \%$ & 1.334 & $2,18 \%$ & 40 & $12,91 \%$ \\
\hline 2005 & 1.911 & $1,79 \%$ & 1.853 & $1,79 \%$ & 58 & $10,68 \%$ \\
\hline 2006 & 2.023 & $1,73 \%$ & 1.964 & $1,76 \%$ & 59 & $10,69 \%$ \\
\hline 2007 & 2.013 & $1,74 \%$ & 1.952 & $1,77 \%$ & 61 & $10,41 \%$ \\
\hline 2008 & 2.009 & $1,74 \%$ & 1.951 & $1,77 \%$ & 58 & $10,68 \%$ \\
\hline 2009 & 2.015 & $1,74 \%$ & 1.960 & $1,76 \%$ & 55 & $10,98 \%$ \\
\hline 2010 & 2.006 & $1,74 \%$ & 1.955 & $1,77 \%$ & 51 & $11,41 \%$ \\
\hline
\end{tabular}

\section{DATOS SECTORIALES}

Si se analizan los datos por sector, a lo largo de todo el periodo 2003-2010, el único sector industrial en el que no aparecen empresas de economía social es el 9 (Industria química y productos farmacéuticos). La intención de este análisis no es la de mostrar diferencias sectoriales, si no la de analizar el peso de la economía social en el conjunto del sector manufacturero español. Por ello consideramos fundamental mantener en la muestra todos los sectores, incluso aquellos en los que no aparecen empresas de economía social. 
Además, no es posible ofrecer información a nivel sectorial, porque los diferentes estratos en este caso no tienen el tamaño suficiente para asegurar la representatividad de la muestra. Por ello, aunque sería muy interesante poder analizar la diferencias entre las distintas ramas industriales, nos hemos limitado a realizar la comparación de las empresas de economía social y el resto de las empresas para el conjunto de la industria manufacturera.

Tabla a.2: Número de empresas por sector (acumulado periodo 2003-2010):

\begin{tabular}{llll}
\hline Sector & $\begin{array}{l}\text { Resto de } \\
\text { empresas }\end{array}$ & $\begin{array}{l}\text { Economía } \\
\text { social }\end{array}$ & Total \\
\hline *1. Industria cárnica & 442 & 9 & 451 \\
*2. Productos alimenticios y tabaco & 1.374 & 43 & 1.417 \\
*3. Bebidas & 283 & 19 & 302 \\
*4. Textiles y confección & 1.021 & 43 & 1.064 \\
*5. Cuero y calzado & 353 & 24 & 377 \\
*6. Industria de la madera & 535 & 8 & 543 \\
*7. Industria del papel & 510 & 3 & 513 \\
*8. Artes gráficas & 703 & 25 & 728 \\
*9. Industria química y productos farmacéuticos & 987 & 0 & 987 \\
*10. Productos de caucho y plástico & 771 & 2 & 773 \\
*11. Productos minerales no metálicos & 1.108 & 30 & 1.138 \\
*12. Metales férreos y no férreos & 496 & 12 & 508 \\
*13. Productos metálicos & 1.828 & 70 & 1.898 \\
*14. Máquinas agrícolas e industriales & 819 & 59 & 878 \\
*15. Productos informáticos, electrónicos y ópticos & 295 & 18 & 313 \\
*16. Maquinaria y material eléctrico & 628 & 10 & 638 \\
*17. Vehículos de motor & 730 & 24 & 754 \\
*18. Otro material de transporte & 342 & 1 & 343 \\
*19. Industria del mueble & 728 & 26 & 754 \\
*20. Otras industrias manufactureras & 351 & 1 & 352 \\
\hline Total & 14.304 & 427 & 14.731 \\
\hline
\end{tabular}

